\title{
Ophthalmic Examinations Completion
}

\section{Status}

National Cancer Institute

\section{Source}

National Cancer Institute. Ophthalmic Examinations Completion Status. NCI Thesaurus.

Code C162335.

A term used to describe the state or condition of the completeness of the ophthalmic examination data. 